This report was prepared as an account of work sponsored by an agency of the United States Government. Neither the United States Government nor any agency thereof, nor any of their employees, makes any warranty, express or implied, or assumes any legal liability or responsibility for the accuracy, completeness, or usefulness of any information, apparatus, product, or process disclosed, or represents that its use would not infringe privately owned rights. Reference herein to any specific commercial product, process, or service by trade name, trademark, manufacturer, or otherwise does not necessarily constitute or imply its endorsement, recommendation, or favoring by the United States Government or any agency thereof. The views and opinions of authors expressed herein do not necessarily state or reflect those of the United States Government or any agency thereof.

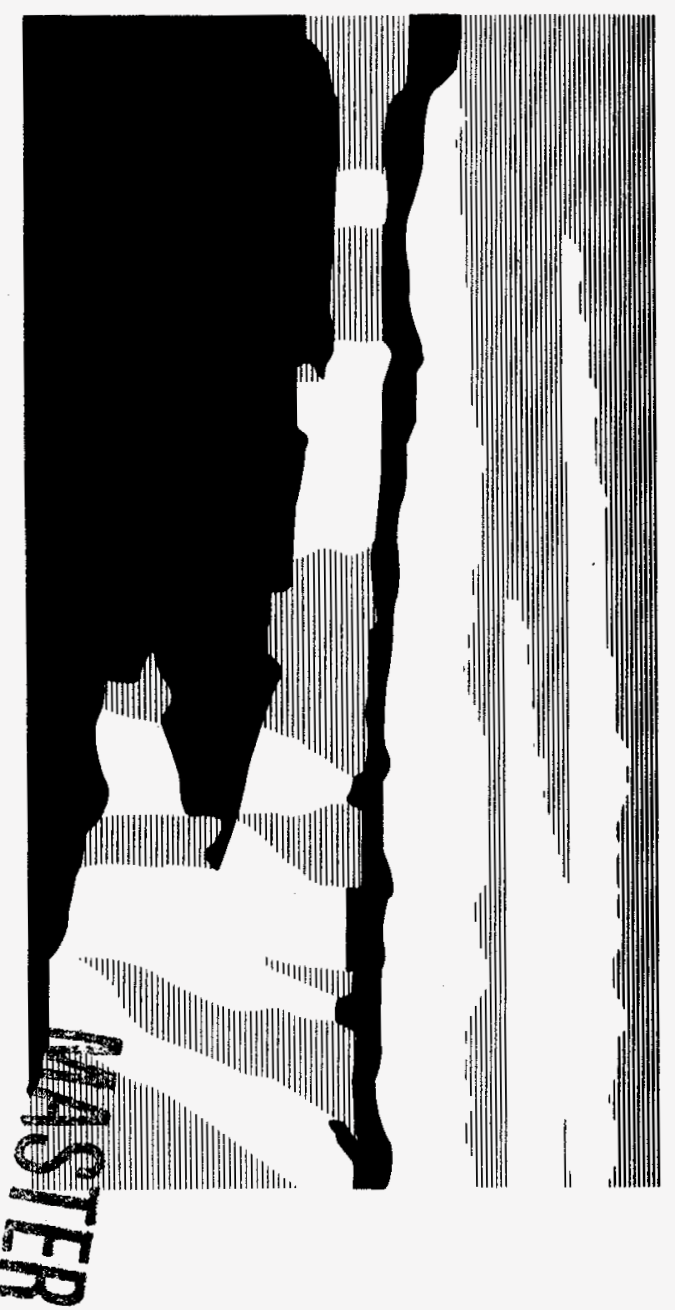

$\frac{n}{5}$ 


\section{DISCLAIMER}

Portions of this document may be illegible in electronic image products. Images are produced from the best available original document. 


\title{
SPALLATION TARGET DESIGN AND INTEGRATION INTO AN ACCELERATOR-BASED TRANSMUTATION SYSTEM
}

\author{
C. A. Beard \\ Los Alamos National Laboratory \\ Los Alamos, NM 87545 \\ (505) 667-0185
}

\begin{abstract}
Spallation target design and system integration is critical for the success of accelerator-based transmutation systems. Issues which must be considered in the design of spallation targets are identified, and representative parametric studies on the system integration of a sample target are given. The results illustrate the importance of a systems-driven target design approach due to the large effects that the target design can have on both the economics and physics performance of the system.

\section{INTRODUCTION}

Accelerator-based systems have been identified as promising candidates for transmuting radioactive waste. Key to the performance (with regard to both physics and economics) of these systems is careful design and integration of a spallation target into the system. Unfortunately, in many accelerator-based transmutation system studies, little attention is given to the spallation target design and integration issues. Rather, spallation targets designed for previous systems and often for other purposes are taken and inserted into the transmutation system with little regard for the overall effects that this approach has on the final system performance. This paper will identify the basic considerations which impact spallation target design and illustrate through parametric analyses the issues which must be dealt with when integrating the target into the overall transmutation system.
\end{abstract}

\section{TARGET FUNCTION AND DESIGN ISSUES}

The function of a spallation target is to convert high-energy ( $\geq 800 \mathrm{MeV}$ ) protons generated by the accelerator to low-energy $(<20 \mathrm{MeV})$ neutrons, and deliver them to the waste-bearing blanket. In performing this function, the target must be cooled sufficiently and designed to reduce risk related to radiological release or other detrimental consequences resulting from off-normal operating conditions ( e.g. loss of target coolant, maladjusted beam distribution, etc. ).

The conversion of high-energy protons to neutrons relies on intranuclear reactions between the incident protons and the nucleons in the target material. Consequently, to maximize neutron production, the target material should have a large number of nucleons per individual nucleus; a high- $Z$ material is preferred. While the protons can interact directly with bound neutrons to eject them from the nucleus, the emitted neutrons tend to be forward peaked and have very high energy ( $20 \mathrm{MeV}$ to the proton energy ) and are poorly used in the blanket, since the slowing-down length is large, even for efficient neutron-moderating materials. Neutrons of more use to a moderating, thermal-neutron blanket are produced through the interaction of a proton with the nucleons in a nucleus in general, thereby leaving the nucleus in an excited state after interacting with the proton. 
Release of excess energy in the excited nucleus occurs by "boiling off" nucleons, a substantial portion of which are neutrons. These neutrons are emitted isotropically with an average energy in the range $1-2 \mathrm{MeV}$.

While maximizing the generation of low-energy neutrons is important to the overall efficiency of the transmutation system, the ultimate target performance depends on its ability to transfer these neutrons to the blanket usefully and efficiently. This efficiency depends on the neutron absorption characteristics of the target material(s) and the volumetric distribution over which the neutrons are generated (i.e., whether the target produces a highly-peaked, intense neutron distribution; or whether the distribution is more evenly distributed; as well as the volume required to achieve maximum neutron production for a given neutron-source distribution). The target absorption characteristics depend not only on intrinsic nuclear parameters, but also on the amount of thermalization that occurs in the target, which is strongly dependent on the type and quantity of coolant used, as well as the target geometry and configuration. Similarly, the neutron-source distribution also depends on the target material (density), coolant fraction ("effective" target density), and geometry. A target design with the overall goal of achieving the required neutron fluxes in the blanket volume with a minimum accelerator beam power, therefore, will have to optimize neutron production, minimize neutron absorption in the target, and maximize neutron leakage to a blanket. Achieving these three goals also requires that the coolant fraction be minimized.

The ability of a spallation target to achieve the functional goals described above depends on three specifications: a) target material, b) target geometry, and c) target heatremoval system. All three specifications are interdependent, however, and this interrelationship must be fully understood before an effective and optimal target design can be realized. Further elaboration of the interdependencies of these specifications and their effects on final target performance can be found in (Beard 1993).

\section{SYSTEM INTEGRATION OF THE SPALLATION TARGET}

For the parametric calculations given below, the target will be assumed to be flowing liquid-lead arranged in a monolithic configuration. The heat is assumed to be removed by transfer from the flowing lead to a secondary coolant in an intermediate heat exchanger. An initial estimate of the required size of the target can be obtained through a simple parameterization of the neutron source emitted based on target size. Figure 1 shows the neutron leakage from an infinitely-long (no bottom leakage) lead target as a function of target radius, and Fig. 2 shows the neutron leakage of a $45-\mathrm{cm}$ radius target as a function of target length. The total leakage curves from Figs. 1 and 2 would indicate an optimal target would be approximately $45 \mathrm{~cm}$ in radius and $60 \mathrm{~cm}$ in length. However, simply maximizing the number of neutrons emitted from the target is not adequate because they only become useful if they enter the blanket region and are absorbed in the fuel. A requirement for bombarding the spallation target with protons is that the protons have an unobstructed pathway to the target surface; this unobstructed pathway also provides a leakage path for generated neutrons to escape without ever entering the blanket. If the target were to be placed at the top of the blanket so that any neutrons leaking from the front face were lost, the target dimensions would optimize solely on radial leakage. The variation of radial leakage with target radius is also shown in Fig. 1. Figure 1 shows that optimizing solely on radial leakage results in a much smaller target radius. Figure 2 also gives the radial leakage as a function of target length. The majority of leakage from a monolithic lead target of this radius $(45 \mathrm{~cm})$ is always in the vertical direction, and for targets of greater than $\sim 15 \mathrm{~cm}$ in length, the main leakage path is off of the front target face. 


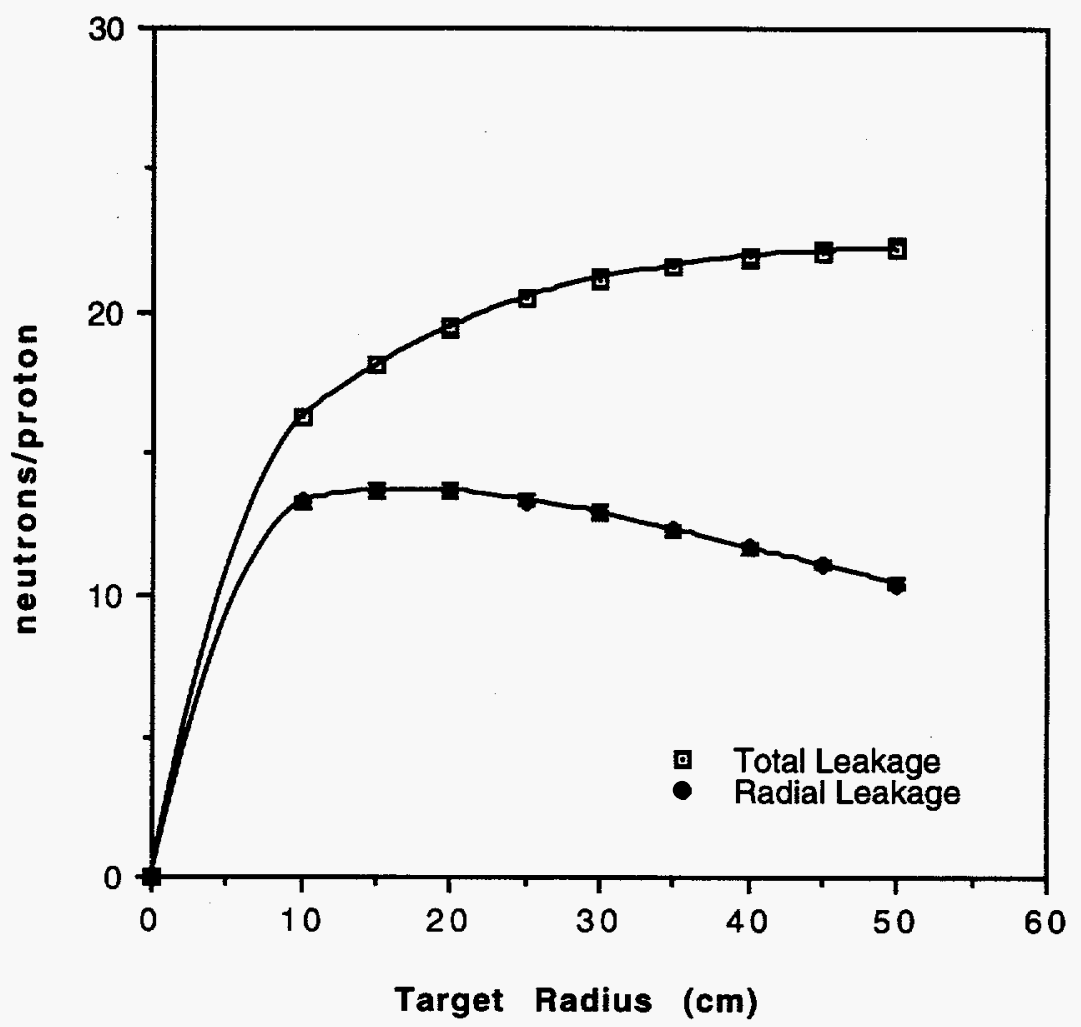

FIGURE 1. Leakage from an infinitely-long (no bottom leakage) lead target.

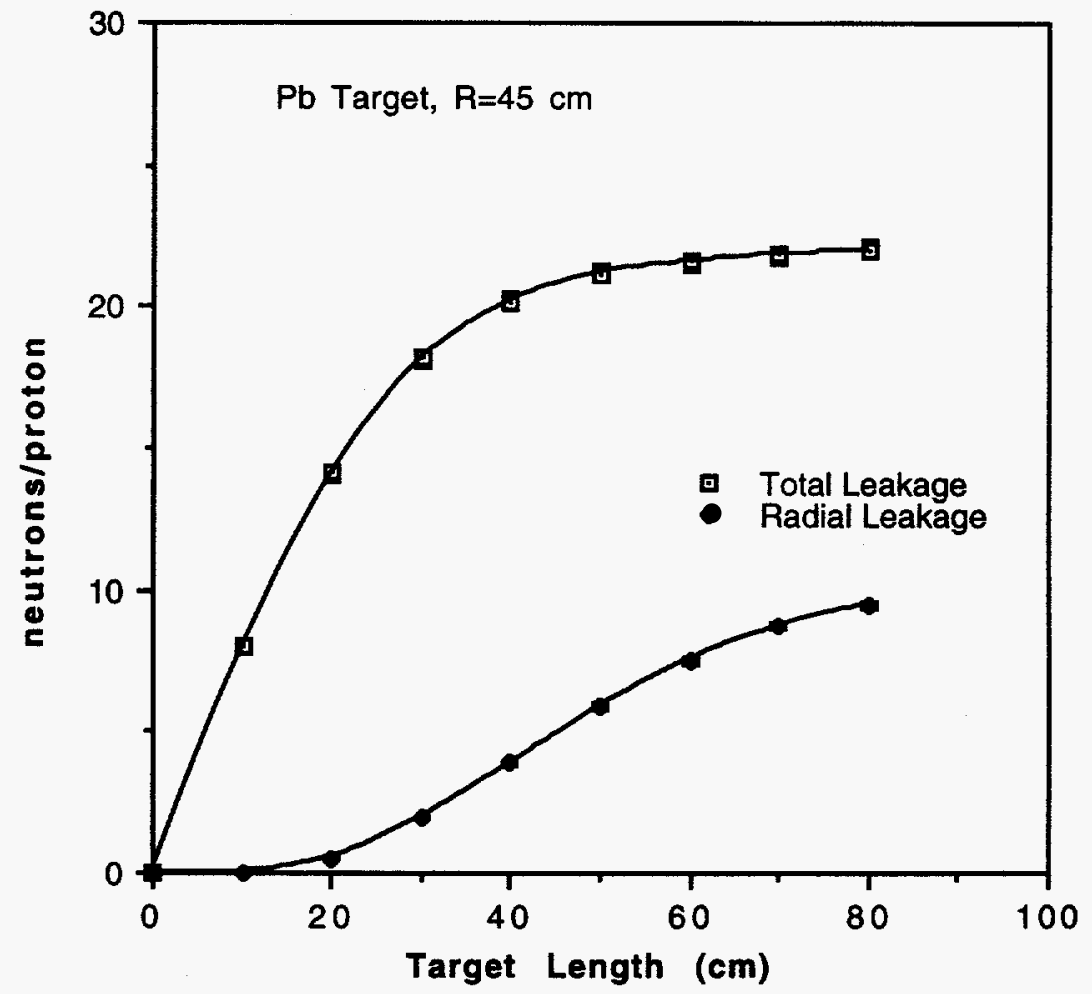

FIGURE 2. Variation of neutron leakage with target length. 
Conversely, if the target is placed at the bottom of the blanket so that any neutrons which leak from the rear of the target are lost, as well as those which leak through the beam pipe, the optimization becomes more complex since it is not just a function of target radius, but of the proton entrance length (distance from top of the blanket to the top of the target) as well. This optimization is shown in Fig. 3. Fig. 3 indicates an optimal target radius of somewhere between $20 \mathrm{~cm}$ and $40 \mathrm{~cm}$, with an entrance length of at least $75 \mathrm{~cm}$. Finally, if the target is moved away from the bottom of the blanket (or made longer), so that neutrons emitted through the rear (of the $60-\mathrm{cm}$ length modeled here) can be reflected back into the useful part of the blanket (or if there is transmutable material located directly behind the target), the optimization changes as shown in Fig. 4. In this case, the useful leakage increases with increasing target radius as long as a substantial entrance length is obtained and the size is not increased to the point that absorption in the lead becomes significant. The optimal dimensions obtained from Fig. 4 are approximately the same as those obtained from the total leakage values in Figs. 1 and 2. Finally, not only does changing target dimensions change the total neutron source emitted, but the distribution of the source as well. Figure 5 shows the axial neutron source distributions for $25-\mathrm{cm}$ and $45-\mathrm{cm}$ radius targets. The target is located at an axial position of $0<\mathrm{z}<60 \mathrm{~cm}$, and the radial leakage is determined through the cylinder encompassing the outer radius of each target. Fig. 5 shows that a smaller target radius produces a much more axially peaked neutron source.

Because the material (in this case, lead) contained in the target will be a waste stream, its volume should be minimized. However, reduction in target volume by changing the internal target geometry causes a change in the total source (as illustrated above). This change in source will then translate into change in accelerator current requirements, which ultimately translates into cost of operation. Fig. 6 shows an optimization calculation for lead mass versus operating (power) costs of the accelerator. It should be noted that the optimization shown in Fig. 6 is very design dependent. In this case, the lead mass is being altered by changing the overall target radius with a set entrance length. The changing beam radius effects to total neutron yield due to changes in proton leakage from the target. This optimization will obviously change depending on the variables being altered. However, the point to be made is that this type of optimization will be required for any specific target design, and arbitrary target design changes cannot be made without first determining the ultimate effects they will have on the system operation.

\section{CONCLUSION}

Design of spallation targets for use in accelerator-based transmutation systems requires careful analysis to insure proper integration into the overall system. The spallation target is the interface between the driving sub-systems of the overall configuration: the accelerator, which typically dominates the capital and operating cost requirements of the system; and the blanket, which determines the ultimate performance of the system as a transmutation device. Determining the effects which the target design has on each of the subsystems is essential if an optimal configuration is to be obtained. Arbitrary use of spallation target designs created for previous transmutation systems, or other uses altogether, typically results in high system-performance penalties. Consequently, in any study evaluating accelerator-based transmutation design concepts, emphasis should be placed on spallation target design, integration, and optimization.

\section{Acknowledgments}

This work was performed under the auspices of the U. S. Department of Energy. 


\section{References}

Beard, C. A., J. J. Buksa, J. W. Davidson, M. G. Houts, R. A. Krakowski, G. F. Niederauer, and L. L. Wang (1993) "An Integrated Test Facility (ITF) for Applications of Accelerator-Driven Transmutation: Preliminary Definition Study and Assessment," Los Alamos National Laboratory document LA-UR-93-4350, Los Alamos, NM.

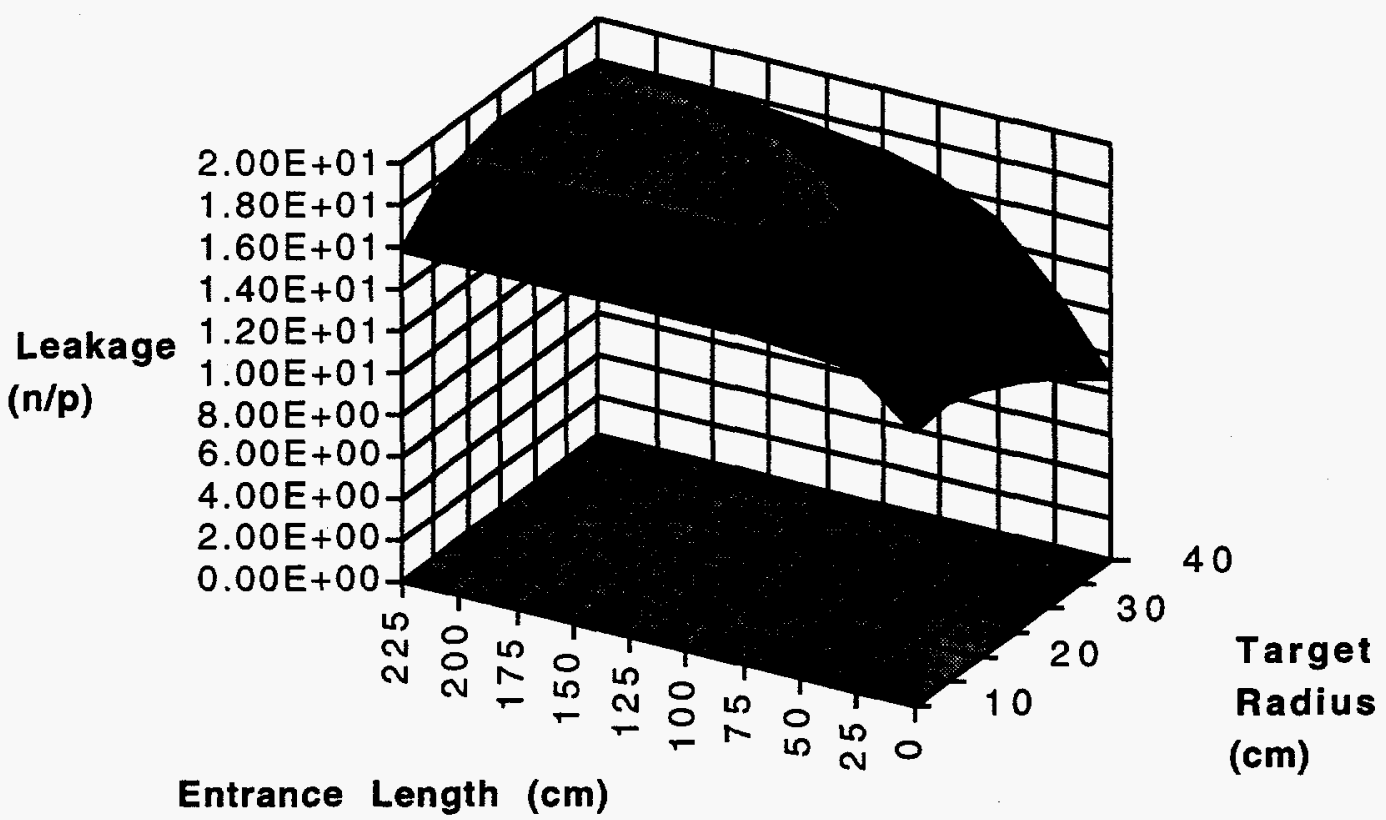
$0.00 E+00$
$2.00 E+00$
$4.00 E+00-$
$6.00 \mathrm{E}+00$
$2.00 \mathrm{E}+00$
$4.00 \mathrm{E}+00$
$6.00 E+00$
$8.00 E+00$
8.00E+00-
$1.00 E+01-$
$1.20 E+01-$
1. $40 E+01-$
$1.00 \mathrm{E}+01$
1. $20 E+01$
$1.40 \mathrm{E}+01$
$1.60 E+01$
$1.60 E+01-$
$1.80 E+01-$
$1.80 E+01$
2.00E+01

FIGURE 3. Parametric variation in radial neutron leakage for a $60-\mathrm{cm}$ long lead target. 


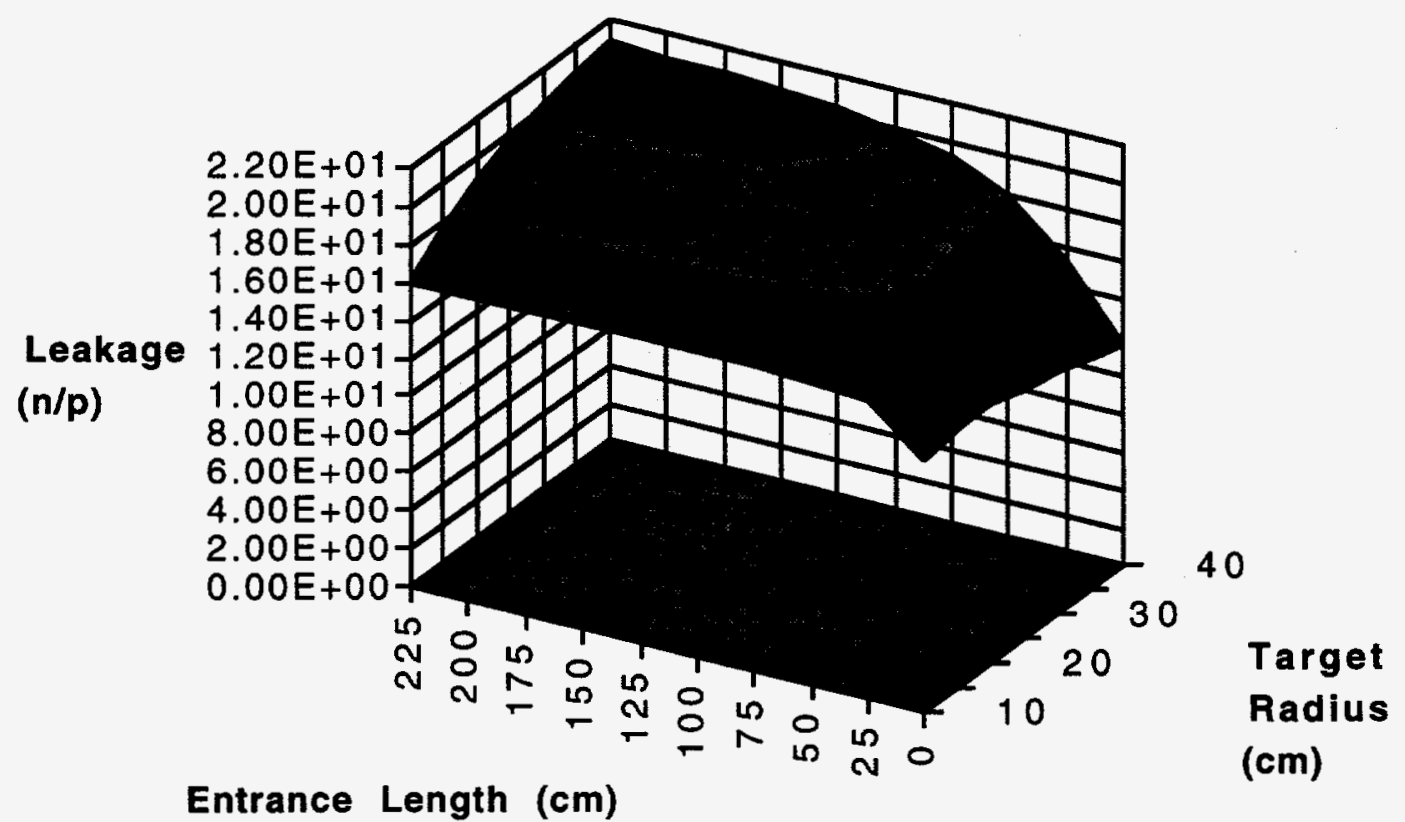

Entrance Length $(\mathrm{cm})$

(cm)

\begin{tabular}{|lccc|}
\hline $\begin{array}{l}0.00 E+00- \\
2.00 E+00\end{array}$ & $4.00 E+00-$ & $4.00 E+00-$ & $0.00 E+00-$ \\
& $4.00 E+00$ & 6.00 & $8.00 E+00$ \\
$8.00 E+00-$ & $1.00 E+01-$ & $1.20 E+01-$ & $1.40 E+01-$ \\
$1.00 E+01$ & $1.20 E+01$ & $1.40 E+01$ & $1.60 E+01$ \\
$1.60 E+01-$ & $1.80 E+01-$ & $2.00 E+01-$ & \\
$1.80 E+01$ & $2.00 E+01$ & $2.20 E+01$ & \\
\hline
\end{tabular}

FIGURE 4. Parametric variation in radial+rear neutron leakage for a $60-\mathrm{cm}$ long lead target. 


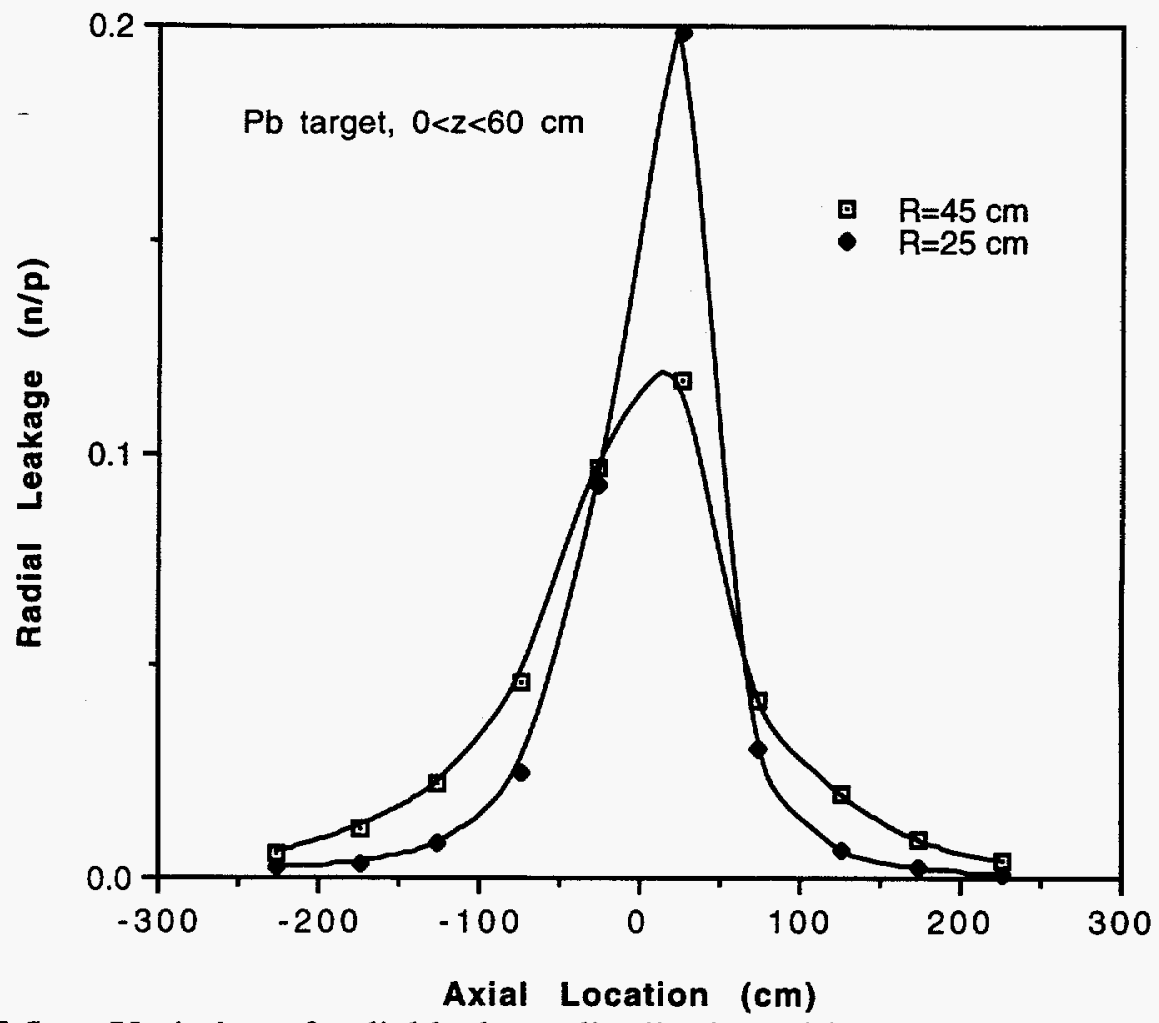

FIGURE 5. Variation of radial leakage distribution with target radius.

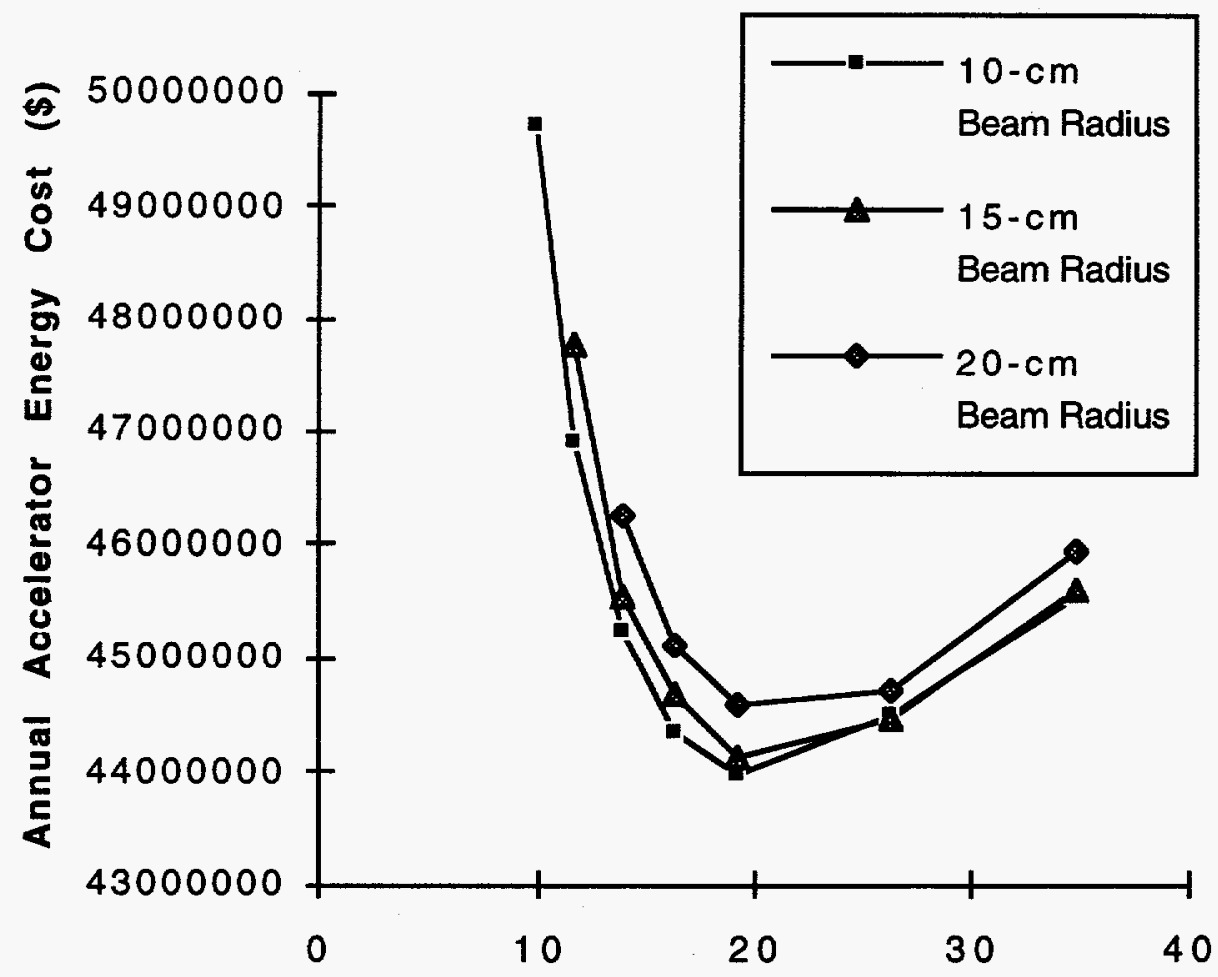

Required Pb Mass in Blanket Region ( MT )

FIGURE 6. Design specific lead mass optimization. 